\title{
Application of Nanoporous Carbon, Extracted from Biomass Combustion Ash, in $\mathrm{CO}_{2}$ Adsorption
}

\author{
Mikhail Gorbounov $^{1}$, Ben Petrovic ${ }^{1}$, Abhishek Lahiri ${ }^{1}$ and Salman Masoudi Soltani ${ }^{1}$ \\ ${ }^{1}$ Department of Chemical Engineering, Brunel University London, Uxbridge UB8 3PH, United \\ Kingdom
}

\begin{abstract}
Bioenergy with Carbon Capture and Storage has been regarded as one of the most prominent technologies in the battle against climate change as stated in the latest Intergovernmental Panel on Climate Change reports. However, solid residues generated during the combustion of biomass pose a separate set of environmental and economic challenges that must be addressed. In order to utilise the full potential of this waste stream, an effective nanoporous carbonaceous adsorbent for $\mathrm{CO}_{2}$ capture has been directly prepared via a simple and low-cost extraction technique from industrial-grade biomass combustion bottom ash generated at a UK power plant. The adsorbent characterisation data (via Scanning Electron Microscopy-Energy Dispersive X-ray Spectroscopy as well as Fourier-Transform Infrared Spectroscopy) agrees well with the $\mathrm{CO}_{2}$ adsorption curves obtained through thermogravimetric analysis (TGA). The TGA results have revealed a $\mathrm{CO}_{2}$ adsorption capacity of 0.73 $\mathrm{mmol} / \mathrm{g}$ at $25{ }^{\circ} \mathrm{C}$ and $1 \mathrm{~atm}$ under a pure $\mathrm{CO}_{2}$ gas stream, thus, proposing a promising and viable route towards in-situ decarbonisation of the biomass combustion sector in the UK via effective waste valorisation.
\end{abstract}

\section{INTRODUCTION}

Climate change has been an ever-present issue for the past few decades. In order to secure a future for generations to come, humanity, as a whole, has to implement drastic measurements to limit the temperature rise to the $2{ }^{\circ} \mathrm{C}$ target as outlined in the Paris Agreement [1]. This goal can be achieved via a significant reduction in global anthropogenic greenhouse gas (GHG) emissions, among which carbon dioxide plays a key role. For instance, the UK government in June 2019 has announced their commitment to reduce (GHGs) emissions to net zero (in comparison to year 1990) by 2050 [2] and in November 2020 have announced "The Ten-Point Plan" which would mobilise $£ 12$ billion of governmental investment [3] thus, accelerating a great deal of research and industrial implementation of Carbon Dioxide Removal (CDR) processes. Out of all the various CDR techniques, Carbon Capture, Utilisation and Storage (CCUS) is regarded as one of the most promising tools for decarbonising industry and the power sector in short- to medium terms thus, playing a crucial role in the uphill battle against climate change. Post-combustion carbon capture (PCCC) is perhaps, the most readily-available option, as it can be retrofitted to the tail end of an emissions source (e.g. fossil-fuel power plants, cement manufacture and etc.), where the capturing media would immobilise the $\mathrm{CO}_{2}$ molecules hence, denying the GHG access to the atmosphere. Afterwards, the collected gas can be transported to a storage site (e.g. depleted oil wells) where it is injected into geological formations for long-term storage. Wide implementation of CCUS would enable the continuation of the utilisation of fossil fuel in the energy sector without the associated harmful environmental (carbon) footprints which, in turn, would allow for a smoother transition into a renewable energy economy by the end of this century. This is especially critical to the developing world where fossil fuels will still play a key part in energy security within the coming decades. However, the shift towards green energy is inevitable. Aligned with this, the use of sustainable biomass to generate heat and power, is projected to increase in the coming years. The combustion of sustainable biomass is a net zero emission process as the $\mathrm{CO}_{2}$ taken up by the plant during its growth is simply put back into the atmosphere. However, coupling the combustion of sustainable biomass for energy generation with PCCC, hence bioenergy and carbon capture and storage (BECCS), translates into a negative emission technology. BECCS is a pivotal technology in $85 \%$ of the scenarios outlined by the Intergovernmental Panel on Climate Change (IPCC) reports to meet climate change targets [4]. On the flip side, biomass combustion is associated with the generation of large quantities of waste ash (6.8\% mean ash yield [5]) - e.g. in the UK approximately 52,000 tonnes of wood ash alone are generated every year [6] and this number is only projected to rise as the country completes the transition away from unabated coal by 2025 [7] due to conversions of coal-fired units into their biomass-combusting counterparts within the UK power sector [8]. There are, however, a range of environmental concerns associated with the disposal of this waste stream (i.e. landfilling). As a result, valorisation of this waste via manufacturing of solid adsorbents for PCCC would not only alleviate the associated landfilling costs, but also provide an alternative to the currently costly PCCC technologies such amine-based absorption processes. This may as well result in a reduction in the total capture cost due to a reduced cost of adsorbent. In this work, industrial-grade biomass combustion bottom ash (BA), has been used to directly extract nanoporous carbonaceous adsorbents for $\mathrm{CO}_{2}$ adsorption. 


\section{MATERials AND Methodology}

Industrial-grade biomass combustion bottom ash was collected from a UK power plant. The waste material was then left to dry in an oven (Fisher Scientific $825 \mathrm{~F}$ ) at $110^{\circ} \mathrm{C}$ for 12 hours. The dried raw BA is shown in Figure 1.

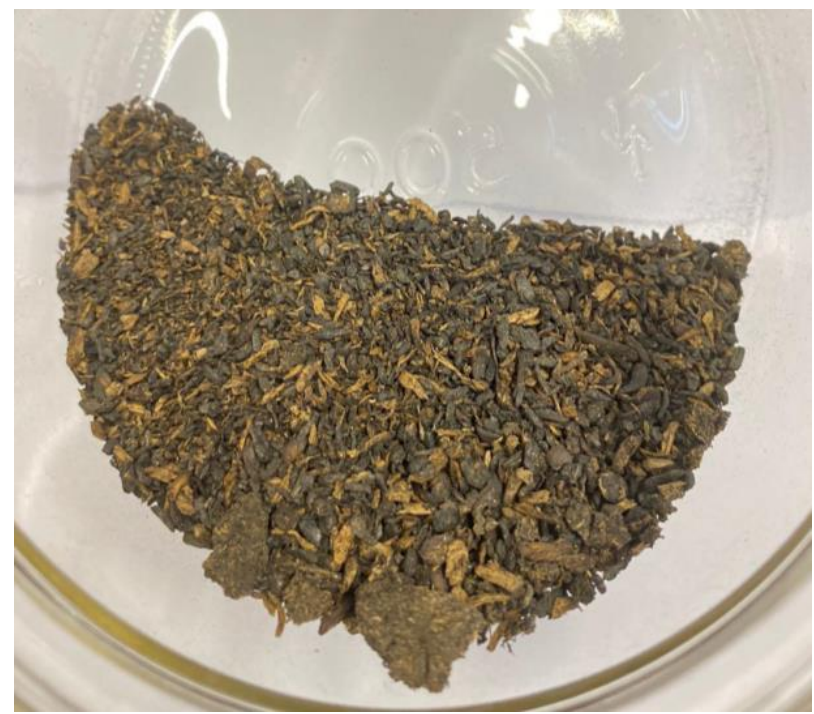

Figure 1. Image of dried raw BA.

The dried sample was next manually ground using a pestle and mortar. The extracted material was then manually agitated in a stack of sieves with varying standard mesh sizes (aperture ranging from $0.38 \mathrm{~mm}$ to $2 \mathrm{~mm}$ ). The larger particulates (consisting mainly of unburnt biomass as seen in Figure 1) were retained by the sieves, whereas the majority of the carbon and ash particles fell through the mesh. In this work, the extract fraction corresponding to a particle size of $<63 \mu \mathrm{m}$ was used for further processing. This fraction was denoted as BA-63-P (Bottom Ash $-63 \mu \mathrm{m}$ - Passed through the sieve) and constituted approximately $30 \%$ of the original dried BA. The obtained carbonaceous material is shown in Figure 2.

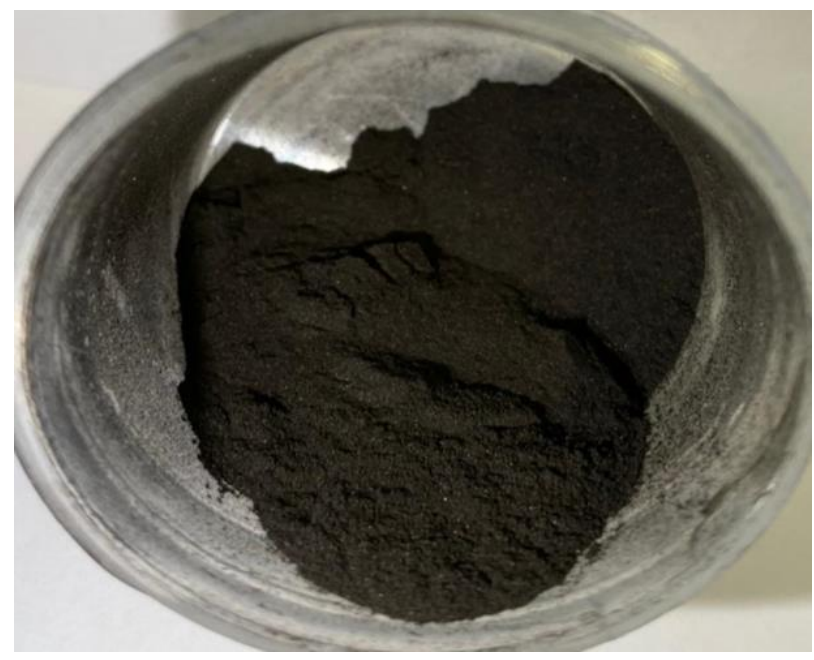

Figure 2. Image of BA-63-P
The properties of BA-63-P were analysed via Scanning Electron Microscopy - Energy Dispersive X-ray Spectroscopy (LEO 1455VP), TGA (Waters Thermal Analysis Instruments SDTQ 600), as well as FourierTransform Infrared Spectroscopy (Perkin Elmer Spectrum One).

\section{RESUlTS AND Discussions}

\section{A. Scanning Electron Microscopy (SEM)}

The surface morphology of the obtained carbon fraction is depicted in Figure 3 and Figure 4.

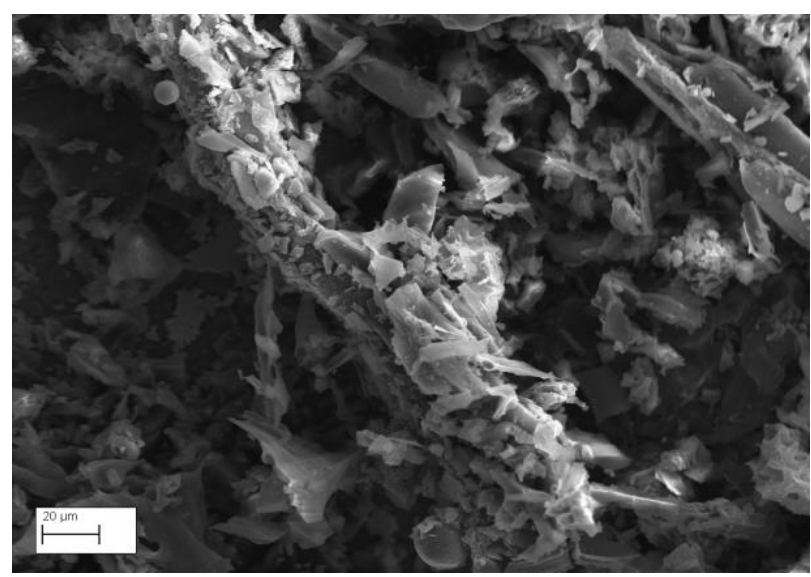

Figure 3. BA-63-P under magnification of $1000 \mathrm{x}$

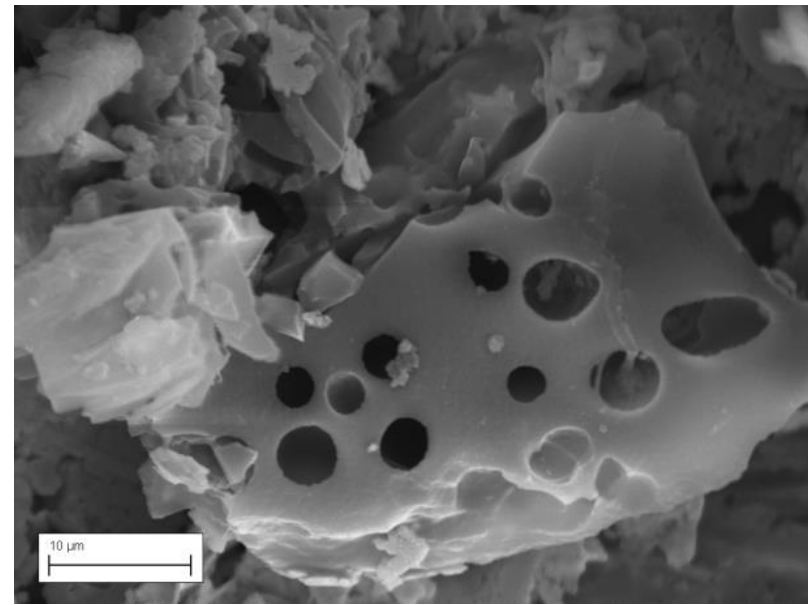

Figure 4. BA-63-P under magnification of 5000x

As seen in Figure 3 and Figure 4, the surface of BA-63$\mathrm{P}$ is porous with random inclusions of spherical particles. These particulates are typical of ashes, and are believed to be of mineral origins (mainly aluminosilicates); their shape and amorphous structure can be attributed to relatively abrupt post-combustion cooling [9], [10].

\section{B. Energy Dispersive X-ray Spectroscopy (EDS)}

BA-63-P has been analysed utilizing EDS and the results are presented in Table I. 
TABLE I. BA-63-P ELEMENTAL COMPOSITION

\begin{tabular}{|c|c|c|}
\hline Element & Weight \% & Atomic \% \\
\hline $\mathrm{C}$ & 75.80 & 81.6 \\
\hline $\mathrm{O}$ & 21.40 & 17.3 \\
\hline $\mathrm{Al}$ & 0.68 & 0.33 \\
\hline $\mathrm{Si}$ & 0.61 & 0.28 \\
\hline $\mathrm{K}$ & 0.52 & 0.17 \\
\hline $\mathrm{Ca}$ & 0.99 & 0.32 \\
\hline
\end{tabular}

The results in Table I are averaged from a number of points analysed across the sample area. Table 1 indicates the high carbon content of the sample.

\section{Fourier-Transform Infrared Spectroscopy (FTIR)}

Infrared spectrum was measured on a Perkin Elmer Spectrum One spectrometer following the standard $\mathrm{KBr}$ method. The measurements were done between 4000 and $450 \mathrm{~cm}^{-1}$ at a $4 \mathrm{~cm}^{-1}$ resolution. It is noteworthy that this technique is not most suitable for identification of inorganic and mineral phases present in the sample. The reason being that the covalent bonds are more prominently displayed on the spectra than ionic bonds. This phenomenon stems from the fact that strong ionic bonds do not vibrate strongly upon infrared absorbance, i.e. they do not change the dipole moment during internal vibrations [11] and therefore, the aforementioned vibrations is hard to detect. The infrared spectrum of BA-63-P is presented in Figure 5.

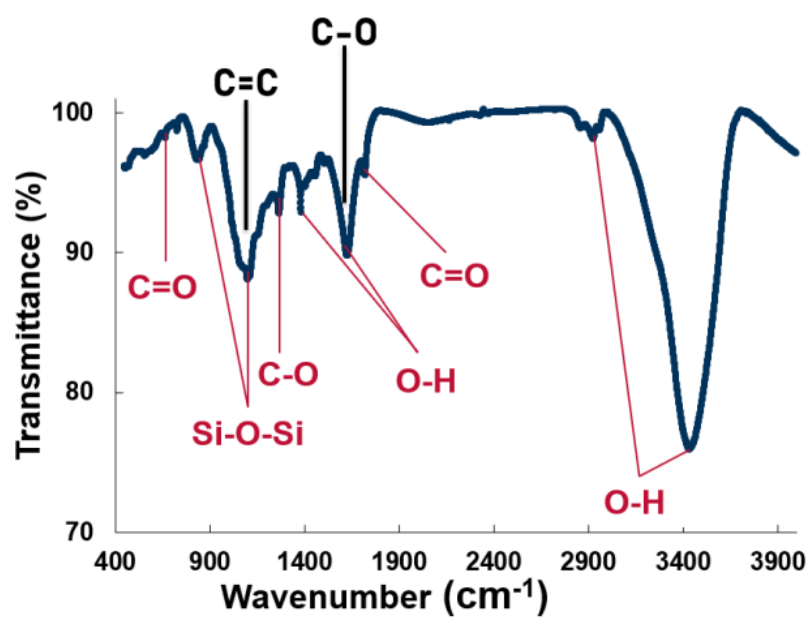

Figure 5. Infrared spectrum of BA-63-P.

The peaks that were identified in the material are as follows (right to left): $3436.56 \mathrm{~cm}^{-1}, 2930.25 \mathrm{~cm}^{-1}, 1722.22$ $\mathrm{cm}^{-1}, 1630.87 \mathrm{~cm}^{-1}, 1384.40 \mathrm{~cm}^{-1}, 1269.00 \mathrm{~cm}^{-1}, 1102.99$ $\mathrm{cm}^{-1}, 840.74 \mathrm{~cm}^{-1}$ and $731.11 \mathrm{~cm}^{-1}$. The two outermost right peaks $\left(3436.56 \mathrm{~cm}^{-1}\right.$ and $\left.2930.25 \mathrm{~cm}^{-1}\right)$ and the peaks at $1630.87 \mathrm{~cm}^{-1}$ and $1384.40 \mathrm{~cm}^{-1}$ are denoted to be the stretching and bending vibrations of the $\mathrm{O}-\mathrm{H}$ bond, respectively [11]-[13]. The band appearing at $1722.22 \mathrm{~cm}^{-1}$ is ascribed to the $\mathrm{C}=\mathrm{O}$ bond. The wavenumbers associated with asymmetric and symmetric $\mathrm{Si}-\mathrm{O}$ vibrations present themselves at $1102.99 \mathrm{~cm}^{-1}$ and $840.74 \mathrm{~cm}^{-1}$, respectively [11], [14]. The lack of a "red shift", or rather the "spot on" placement of the wavenumbers, may point out to the presence of aluminium-free silicon oxide species. The band at $731.11 \mathrm{~cm}^{-1}$ may be associated with the double bond between carbon and oxygen atoms in a carbonate group [15]. The wavelength at $1269 \mathrm{~cm}^{-1}$ may also be attributed C-O bond and $\mathrm{C}-\mathrm{O}-\mathrm{H}$ groups [16], which could be found as carbonated species in ash, or as the abovementioned functional groups on the surface of the particles. However, the spectrum can also be interpreted differently if, for instance, re-ascribing the $2930.25 \mathrm{~cm}^{-1}$ peak to vibrations corresponding to aliphatic $\mathrm{C}-\mathrm{H}$, where the wavenumber at $1630.87 \mathrm{~cm}^{-1}$ might suggest the $\mathrm{C}=\mathrm{C}$ bond found in graphite, graphene and graphene oxides [17]. In addition, the bond just over $1100 \mathrm{~cm}^{-1}$ can be re-assigned to a $\mathrm{C}-\mathrm{O}$ bond from graphene oxides [17]. Nevertheless, either of the two lines of thought explained above point out the presence of unburnt carbon alongside ash impurities.

\section{Proximate Analysis}

In order to quantify the amount of impurities as well as the fixed and the volatile organic carbon (VOC) present in the sample, proximate analysis was employed [18]. The results are presented in Table II.

TABLE II. BA-63-P PROXIMATE ANALYSIS RESUlts.

\begin{tabular}{|c|c|c|c|}
\hline $\begin{array}{c}\text { Moisture, } \\
\text { wt\% }\end{array}$ & $\begin{array}{c}\text { VOC, } \\
\text { wt\% }\end{array}$ & $\begin{array}{c}\text { Fixed C, } \\
\text { wt\% }\end{array}$ & $\begin{array}{c}\text { Ash, } \\
\text { wt\% }\end{array}$ \\
\hline 6 & 35 & 52 & 7 \\
\hline
\end{tabular}

The proximate analysis results also confirm the carbonrich nature of the sample, with minor amorphous aluminosilicate ash inclusions. The data indicates the successful extraction of unburnt carbon from BA in this work.

\section{E. Adsorptive properties and Characteristics}

The adsorptive properties of BA-63-P have been evaluated using a TGA apparatus. Effective adsorbents suitable for post-combustion carbon capture have been reported to have an uptake capacity of about $2 \mathrm{mmol}_{\mathrm{CO} 2} /$ $\mathrm{g}_{\text {sorbent }}$ at $1 \mathrm{~atm}$ and $25^{\circ} \mathrm{C}$ under a flow of pure $\mathrm{CO}_{2}$ [19]. Our directly extracted carbonaceous adsorbent (without any post-activation) shows a promising uptake capacity of 0.73 $\mathrm{mmol} / \mathrm{g}(32.2 \mathrm{mg} / \mathrm{g})$ of carbon dioxide under the above experimental conditions.

\section{CONCLUSION}

The results in this work show a potentially feasible route for economical extraction of effective carbonaceous adsorbents from waste BA. The synthesised adsorbent indicated a relatively good $\mathrm{CO}_{2}$ uptake capacity of 0.73 $\mathrm{mmol}_{\mathrm{CO} 2} / \mathrm{g}_{\text {adsorbent }}$ without any post activation. Within the next steps, further studies will be conducted in order to improve the adsorption capacity of the adsorbent and to evaluate the adsorption kinetics under various operating conditions that better resemble that of an actual flue gas. Such knowledge will be invaluable to the understanding of the capital and operational expenses (CAPEX/OPEX) of the process. 


\section{ACKNOWLEDGMENT}

This work has been funded by the UK Carbon Capture and Storage Research Centre (EP/P026214/1) through the flexible funded research programme "Biomass Combustion Ash in Carbon Capture". The UKCCSRC is supported by the Engineering and Physical Sciences Research Council (EPSRC), UK, as part of the UKRI Energy Programme. The authors are grateful to the Research Centre for providing this funding. The authors would also like to acknowledge Brunel Research Initiative and Enterprise Fund (BRIEF) to support this work.

\section{REFERENCES}

[1] United Nations, "Summary of the Paris Agreement," United Nations Framew. Conv. Clim. Chang., pp. 27-52, 2015.

[2] B. Petrovic, M. Gorbounov, and S. Masoudi Soltani, "Influence of surface modification on selective $\mathrm{CO}<\mathrm{inf}>2</ \mathrm{inf}>$ adsorption: A technical review on mechanisms and methods,"

Microporous Mesoporous Mater., vol. 312, 2021.

[3] UK Government, “The Ten Point Plan for a Green Industrial Revolution." [Online]. Available: https://www.gov.uk/government/publications/theten-point-plan-for-a-green-industrialrevolution/title. [Accessed: 03-Jun-2021].

[4] IPCC, "Climate Change 2014," 2014.

[5] S. V. Vassilev, D. Baxter, L. K. Andersen, and C. G. Vassileva, "An overview of the composition and application of biomass ash. Part 1. Phasemineral and chemical composition and classification," Fuel, vol. 105, pp. 40-76, 2013.

[6] É. J. M. Bastien, "Towards Circular Economy : Wood ash management for biomass CHP plants in the UK," KTH Royal Institute of Technology, 2020.

[7] BEIS, "IMPLEMENTING THE END OF UNABATED COAL BY 2025 Government response to unabated coal closure consultation," 2018.

[8] BEIS, "Energy Trends UK, October to December 2020," 2021.

[9] B. G. Kutchko and A. G. Kim, "Fly ash characterization by SEM-EDS," Fuel, vol. 85, no. 17-18, pp. 2537-2544, 2006.

[10] D. Mainganye, T. Ojumu, and L. Petrik, "Synthesis of Zeolites Na-P1 from South African Coal Fly Ash: Effect of Impeller Design and Agitation," Materials (Basel)., vol. 6, no. 5, pp. 2074-2089, May 2013.

[11] W. Mozgawa, M. Król, J. Dyczek, and J. Deja, "Investigation of the coal fly ashes using IR spectroscopy," Spectrochim. Acta - Part A Mol. Biomol. Spectrosc., vol. 132, pp. 889-894, 2014.

[12] M. Gómez et al., "Development of mesoporous materials from biomass ash with future applications as adsorbent materials," Microporous Mesoporous Mater., vol. 299, no. January, 2020.
[13] P. Raizada, P. Shandilya, P. Singh, and P. Thakur, "Solar light-facilitated oxytetracycline removal from the aqueous phase utilizing a $\mathrm{H} 2 \mathrm{O} 2$ /ZnWO 4 /CaO catalytic system,"J. Taibah Univ. Sci., vol. 11, no. 5, pp. 689-699, 2017.

[14] M. R. Derrick, D. Stulik, and J. M. Landry, Infrared Spectroscopy in Conservation Science. Los Angeles, 1999.

[15] Y. Chen, C. Zou, M. Mastalerz, S. Hu, C. Gasaway, and X. Tao, "Applications of microfourier transform infrared spectroscopy (FTIR) in the geological sciences-A Review," Int. J. Mol. Sci., vol. 16, no. 12, pp. 30223-30250, 2015.

[16] X. Feng, Q. Zhuang W, C. P, and J. Tang Y, Microbial Systems Biology. Methods in Molecular Biology (Methods and Protocols). 2012.

[17] Sudesh, N. Kumar, S. Das, C. Bernhard, and G. D. Varma, "Effect of graphene oxide doping on superconducting properties of bulk MgB2," Supercond. Sci. Technol., vol. 26, no. 9, 2013.

[18] ASTM, "ASTM D3172-13, Standard Practice for Proximate Analysis of Coal and Coke," 2013. [Online]. Available: https://www.astm.org/Standards/D3172.htm.

[19] S. H. Liu, Y. C. Lin, Y. C. Chien, and H. R. Hyu, "Adsorption of $\mathrm{Co} 2$ from flue gas streams by a highly efficient and stable aminosilica adsorbent," J. Air Waste Manag. Assoc., vol. 61, no. 2, pp. 226-233, 2011. 